\title{
Toy Models of Universe with an Effective Varying $\Lambda$-Term in Lyra Manifold
}

\author{
Martiros Khurshudyan \\ Max Planck Institute of Colloids and Interfaces, Potsdam-Golm Science Park, Am Muhlenberg 1 OT Golm, 14476 Potsdam, Germany \\ Correspondence should be addressed to Martiros Khurshudyan; khurshudyan@yandex.ru
}

Received 6 October 2014; Accepted 21 December 2014

Academic Editor: Sally Seidel

Copyright ( 2015 Martiros Khurshudyan. This is an open access article distributed under the Creative Commons Attribution License, which permits unrestricted use, distribution, and reproduction in any medium, provided the original work is properly cited. The publication of this article was funded by SCOAP S $^{3}$

\begin{abstract}
Research on the accelerated expansion of our Universe captures a lot of attention. The dark energy (DE) is a way to explain it. In this paper we will consider scalar field quintessence DE with $\omega_{\mathrm{DE}}>-1 \mathrm{EoS}$, where the dynamics of the DE models related to the dynamics of the scalar field. We are interested in the study of the behavior of the Universe in the presence of interacting quintessence DE models in Lyra manifold with a varying $\Lambda(t)$. In a considered framework we also would like to propose a new form for $\Lambda(t)$. We found that the models correspond to the transit Universe, which will enter the accelerated expansion phase and will remain there with a constant deceleration parameter $q$. We found also that the $\Lambda(t)$ is a decreasing function which takes a small positive value with $\Omega_{m} \neq 0$ and $\Omega_{\mathrm{Q}} \rightarrow$ const dominating $\Omega_{m}$ in the old Universe. Observational constraints are applied and causality issue via $C_{S}^{2}$ is discussed as a possible way to either reject or accept the models.
\end{abstract}

\section{Introduction}

Analysis of the observational data shows that our Universe for later stages of the evolution indicates accelerated expansion [1-3]. According to the observational data we know that in the Universe one of the main components is dark energy and its negative pressure (positive energy density) has enough power to work against gravity and provide accelerated expansion of the Universe. To have a balance in the Universe the second component known as dark matter (DM) is considered, which is responsible for the completely other phenomenon known as structure formation. According to different estimations the DE occupies about $73 \%$ of the energy of our Universe, while $\mathrm{DM}$ is about $23 \%$, and usual baryonic matter is about $4 \%$. The surveys on clusters of galaxies showed that the density of matter is very much less than critical density [4]; observations of cosmic microwave background (CMB) anisotropies indicate that the Universe is at and the total energy density is very close to the critical $\Omega_{\text {tot }} \simeq 1$ [5]. However, an interesting question is how does our Universe approach the flatness observed today. It is interesting to mention theoretical investigations which show that our Universe can approach the isotropy monotonically even in the presence of an anisotropic fluid; moreover the anisotropy of the fluid also isotropizes at later times for the accelerating expansion of the Universe. For instance, such scenarios were obtained in [6]. Deeper analysis reveals that in cosmological models the Universe can achieve the slightly anisotropic geometry in spite of the inflation. Therefore, we can classify two groups of the models depending on whether this happens as it is discussed in [6]. Various interesting papers and cosmological models working with the anisotropy of the Universe, anisotropy of the DE, and vector DE models exist in literature and we will refer our readers to [6] and references therein, because discussion of such cosmology is out of the main goal in this work. The simple model for the DE is the cosmological constant with two problems called fine-tuning and coincidence [7]. These problems have opened ways for alternative models for the DE including dynamical forms of it, as a variable cosmological constant $[8,9], k$-essence model $[10,11]$, and Chaplygin gas models [12-28] to mention a few. In recent times it was shown that certain type of interactions between DE and DM also could solve the mentioned problems. To solve dark energy problem, on the other hand, we can modify the left-hand 
side of Einstein equations and obtain theories such as $f(R)$ theory of the gravity [29-38]. Modifications of these types provide a natural way to explain the origin of the dark energy. But such theories with different forms of modifications still should pass experimental tests, because they contain ghosts, finite-time future singularities, and so on, which is the base of other theoretical problems. One of the well studied DE models is a quintessence model [39-47], which is a scalar field model described by a scalar field $\phi$ and $V(\phi)$ potential and it is the simplest scalar field scenario without having theoretical problems such as the appearance of ghosts and Laplacian instabilities. Energy density and pressure of quintessence DE are given as

$$
\begin{aligned}
& \rho_{\mathrm{Q}}=\frac{1}{2} \dot{\phi}^{2}+V(\phi), \\
& P_{\mathrm{Q}}=\frac{1}{2} \dot{\phi}^{2}-V(\phi) .
\end{aligned}
$$

Our model of the Universe contains an effective twocomponent fluid with an effective energy density and a pressure given as

$$
\begin{aligned}
& \rho=\rho_{\mathrm{Q}}+\rho_{b}, \\
& P=P_{\mathrm{Q}}+P_{b},
\end{aligned}
$$

where $\rho_{b}$ and $P_{b}$ are the energy density and the pressure of a barotropic fluid, respectively, which will model the DM in our Universe with a $P_{b}=\omega_{b} \rho_{b}$ EoS equation. The last assumption concerning the energy density and the pressure of the effective fluid can work particularly for the old and large scale Universe, where quantum and nonequilibrium effects are not considered. Whether the last assumption works in the early Universe is an open question, because, for the early Universe with high energy, small scales quantum effects can have unexpected effects and how the situation should be modified is not clear yet. As long as we have other conceptual problems, for instance, we do not know how correctly we can model the content of the early Universe. The question of how an interaction between the fluid components arose is not answered yet as well. One of the assumptions concerning the interaction between components is probably the same origin of the DE and the DM; however, this hypothesis is not a satisfactory approach to the problem. Despite the fact that the question is not answered yet, we continue the consideration of the different interactions; moreover we continue also performing some modifications based mainly on phenomenology. In literature we can find different cosmological models admitting different forms of the interaction $Q$ between the fluid components of the Universe. One of them with a general form is

$$
Q=q^{\kappa}\left(3 H \sum_{i} b_{i} \rho_{i}+\sum_{i} \gamma_{i} \dot{\rho}_{i}\right),
$$

where $b_{i}$ and $\gamma_{i}$ are positive constants, $q$ is the deceleration parameter, and $\kappa$ is a constant. $\kappa=1$ will correspond to a signchangeable interaction [48]. The typical value of the constants is about $0.01 \div 0.03$. A phenomenological modification of $Q$ can include a possibility with $b_{i}$ or $\gamma_{i}$ to be functions of a cosmological parameter, that is, to consider $b(t)_{i}$ and $\gamma(t)_{i}$. In this study we consider a quintessence $\mathrm{DE}$ with exponential self-interacting potential $V(\phi)$ of the form $[49,50]$

$$
V(\phi)=V_{0} e^{[-\alpha \phi]},
$$

interacting with a barotropic fluid via

$$
Q=3 H b \rho+\gamma \dot{\rho},
$$

where $V_{0}, \alpha, b$, and $\gamma$ are constants, obtained from (5) with $\kappa=0$. The dynamics defined in Lyra manifold with a varying $\Lambda(t)$ will be considered instead of the dynamics provided by GR. This work will differ from the other similar works in literature by the models with a new form for the $\Lambda(t)$. We know that a $\Lambda(t)$ should be a decreasing function over time and has a small positive value in recent Universe. It will be seen that the proposed $\Lambda(t)$, which is a function from the Hubble parameter $H$, scalar field $\phi$, and self-interacting potential $V(\phi)$ like [51]

$$
\Lambda(t)=H^{2} \phi^{-2}+\delta V(\phi),
$$

can achieve the desirable behavior.

The paper is organized as follows. In Section 2 we review the modified field equations. In Section 3 we analyse the models. In Section 4 we discuss the causality issue and observational constraints on the models. The last section includes conclusion and discussion about the cosmological consequence provided by the suggested cosmological models.

\section{The Field Equations}

Lyra geometry is an example of scalar tensor theory and one of the modifications of GR suggested by Lyra as a modification of Riemannian geometry [52]. In this modification the Weyl's gauge is modified. Field equations that constructed an analogue of the Einstein field equations based on Lyra's geometry can be written as $[53,54]$

$$
R_{\mu \nu}-\frac{1}{2} g_{\mu \nu} R+\frac{3}{2} \psi_{\mu} \psi_{\nu}-\frac{3}{4} g_{\mu \nu} \psi^{\alpha} \psi_{\alpha}=T_{\mu \nu} .
$$

It was pointed out that the constant displacement field $\psi_{\alpha}$ of this theory can be interpreted as a cosmological constant $\Lambda$ in the normal relativistic treatment [55]. We are interested in the other modification of the field equations which contain varying cosmological constant $\Lambda(t)$ and which can be written as [56]

$$
R_{\mu \nu}-\frac{1}{2} g_{\mu \nu} R-\Lambda g_{\mu \nu}+\frac{3}{2} \psi_{\mu} \psi_{\nu}-\frac{3}{4} g_{\mu \nu} \psi^{\alpha} \psi_{\alpha}=T_{\mu \nu} .
$$

Considering the content of the Universe to be a perfect fluid, we have

$$
T_{\mu \nu}=(\rho+P) u_{\mu} u_{\nu}-P g_{\mu \nu},
$$


where $u_{\mu}=(1,0,0,0)$ is a 4 -velocity of the comoving observer, satisfying $u_{\mu} u^{\mu}=1$. Let $\psi_{\mu}$ be a time-like vector field of displacement,

$$
\psi_{\mu}=\left(\frac{2}{\sqrt{3}} \beta(t), 0,0,0\right),
$$

where $\beta=\beta(t)$ is a function of time alone, and the factor $2 / \sqrt{3}$ is substituted in order to simplify the writing of all the following equations. By using FRW metric for a flat Universe,

$$
d s^{2}=-d t^{2}+a(t)^{2}\left(d r^{2}+r^{2} d \Omega^{2}\right),
$$

field equations can be reduced to the following Friedmann equations:

$$
\begin{gathered}
3 H^{2}-\beta^{2}=\rho+\Lambda(t), \\
2 \dot{H}+3 H^{2}+\beta^{2}=-P+\Lambda(t),
\end{gathered}
$$

where $H=\dot{a} / a$ is the Hubble parameter, and an overdot stands for differentiation with respect to cosmic time $t, d \Omega^{2}=$ $d \theta^{2}+\sin ^{2} \theta d \phi^{2}$, and $a(t)$ represents the scale factor. The $\theta$ and $\phi$ parameters are the usual azimuthal and polar angles of spherical coordinates, with $0 \leq \theta \leq \pi$ and $0 \leq \phi<2 \pi$. The coordinates $(t, r, \theta, \phi)$ are called comoving coordinates.

The continuity equation reads as

$$
\dot{\rho}+\dot{\Lambda}+2 \beta \dot{\beta}+3 H\left(\rho+P+2 \beta^{2}\right)=0 .
$$

With an assumption that

$$
\dot{\rho}+3 H(\rho+P)=0 .
$$

Equation (13) will give a link between $\Lambda(t)$ and $\beta(t)$ of the following form:

$$
\dot{\Lambda}+2 \beta \dot{\beta}+6 H \beta^{2}=0 .
$$

To introduce an interaction between the $\mathrm{DE}$ and the DM, we should mathematically split (14) and consider the following two equations:

$$
\begin{aligned}
& \dot{\rho}_{\mathrm{DM}}+3 H\left(\rho_{\mathrm{DM}}+P_{\mathrm{DM}}\right)=\mathrm{Q}, \\
& \dot{\rho}_{\mathrm{DE}}+3 H\left(\rho_{\mathrm{DE}}+P_{\mathrm{DE}}\right)=-Q .
\end{aligned}
$$

Cosmological parameters of our interest are EoS parameters of each fluid component $\omega_{i}=P_{i} / \rho_{i}$, EoS parameter of composed fluid

$$
\omega_{\text {tot }}=\frac{P_{m}+P_{\Lambda}}{\rho_{m}+\rho_{\Lambda}},
$$

and deceleration parameter $q$, which can be written as

$$
q=\frac{1}{2}\left(1+3 \frac{P}{\rho}\right) \text {. }
$$

To study the causality issue we need also the behavior of the square of the sound speed with the widespread accepted opinion with the following constraint on it:

$$
0 \leq\left(C_{S}^{2} \equiv \frac{\partial P}{\partial \rho}\right) \leq 1,
$$

to accept the cosmological models. However, the last opinion also can be challenged. Constant cosmological constant $\Lambda$ produces models of the Universe where $\beta(t) \rightarrow 0$, with $a(t) \rightarrow \infty$, because we have the following form for the $\beta(t)$ :

$$
\beta=\beta_{0} a^{-3},
$$

as a solution of (15), where $a(t)$ is the scale factor and $\beta_{0}$ is the integration constant, which means that for the very large scale Universe the dynamics again will correspond to the dynamics given by GR. In the next section we start the analysis of the models.

\section{The Model and the Cosmological Parameters}

The cosmological model with the varying cosmological constant $\Lambda(t)$ and the potential given by (6) and (4) will determine the behavior of the $\beta(t)$ as

$$
2 \beta \dot{\beta}+6 H \beta^{2}+2 H \dot{H} \phi^{-2}-2 H^{2} \phi^{-3} \dot{\phi}-\delta \alpha e^{[-\alpha \phi]} \dot{\phi}=0 .
$$

Having the interaction $Q=3 H b \rho+\gamma \dot{\rho}$ between the fluid components gives us a transit Universe (i.e., a transition to the Universe with $q>0$ to the Universe with $q<0$, where the Hubble parameter $H$ is a decreasing function (Figure 1) which enters the ever accelerating expansion phase, where $\Omega_{m}, \beta(t)$ with $\Lambda(t)$ are small constants and constantly exist in the old Universe, while $\Omega_{\mathrm{Q}} \approx 0.74$ and dominates to the $\mathrm{DM}$ (Figure 2). The behavior of the $\beta(t)$ clearly shows that the dynamics of the old Universe will be described by the theory differing from GR, but the proof of this fact could not appear in a simple way from the observations due to the very small value of $\beta(t)$. In such Universe the dynamics of the energy densities of the fluid components will be governed according to the two following equations:

$$
\begin{aligned}
& (1-\gamma) \dot{\rho}_{b}+3 H\left(1+\omega_{b}-b\right) \rho_{b}=3 H b \rho_{\mathrm{Q}}+\gamma \dot{\rho}_{\mathrm{Q}}, \\
& (1+\gamma) \dot{\rho}_{\mathrm{Q}}+3 H\left(1+\omega_{\mathrm{Q}}+b\right) \rho_{\mathrm{Q}}=-3 H b \rho_{b}-\gamma \dot{\rho}_{b} .
\end{aligned}
$$

Including the interaction under consideration into the model and having a strong interaction described by $b>0.03$ and $\gamma>0.03$, we see that we can increase values of $\beta(t)$ and $\Lambda(t)$ for the later stages of the evolution. The behavior of the $\omega_{\text {tot }}$ indicates that our Universe will start its evolution with the fluid with a positive $\omega_{\text {tot }}$; then we will have a transition to the Universe where $\omega_{\text {tot }}<0$. The effective fluid and DE are quintessence fluids with $\omega_{\text {tot }}>-1$ and $\omega_{\mathrm{Q}}>-1$. We see that increasing $b$ and $\gamma$ (and $\delta$ ) will decrease $\omega_{\mathrm{Q}}$ and $\omega_{\text {tot }}$ in the accelerated expansion phase of the old Universe (Figure 3). The last two cosmological parameters are not strongly dependent on the EoS parameter of the barotropic fluid, and with big accuracy the fact that changes in $\omega_{b}$ will not change $\omega_{\text {tot }}$ and $\omega_{\mathrm{Q}}$ could be accepted. It is also interesting to understand the behavior of the model for different forms of the interactions. We investigated the behavior of the Universe 


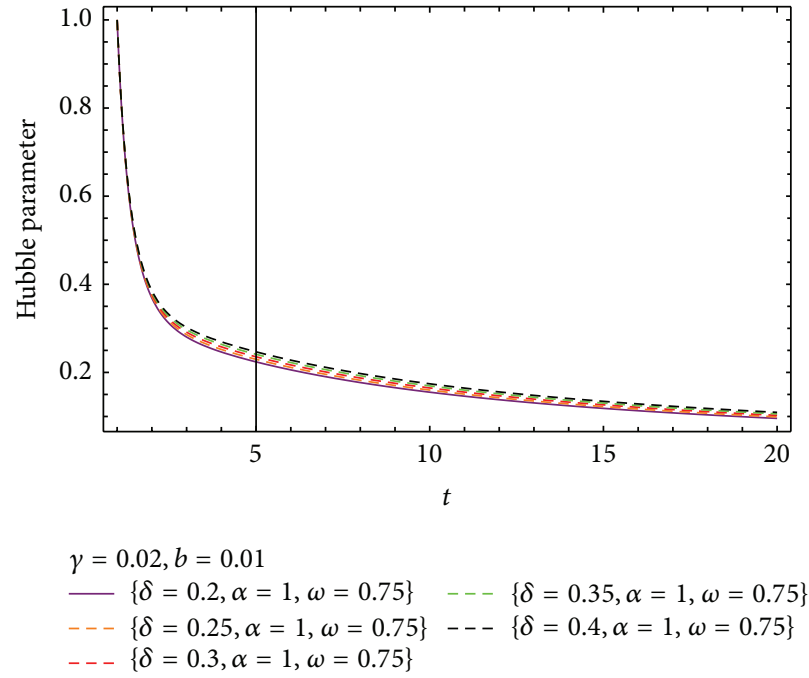

(a)

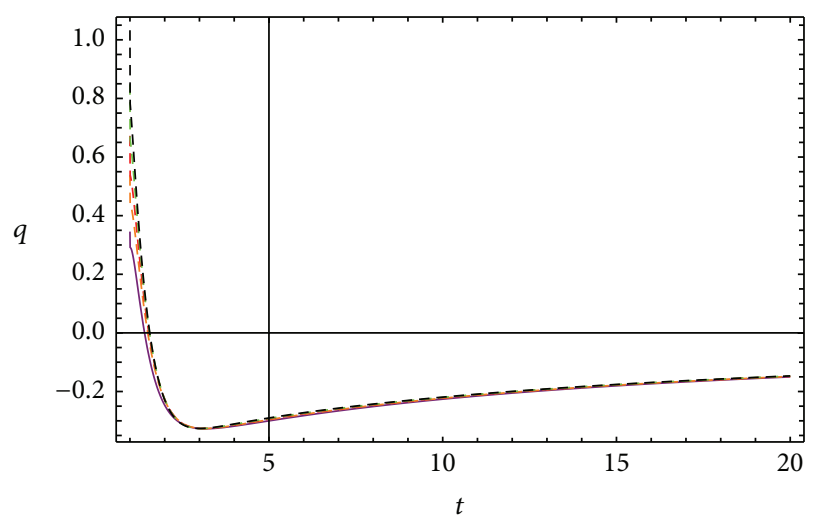

$$
\begin{array}{ll}
\gamma=0.02, b=0.01 & \\
-\{\delta=1.5, \alpha=1, \omega=0\} & --\{\delta=1.5, \alpha=1, \omega=0.75\} \\
---\{\delta=1.5, \alpha=1, \omega=0.3\} & ---\{\delta=1.5, \alpha=1, \omega=1\} \\
---\{\delta=1.5, \alpha=1, \omega=0.5\} &
\end{array}
$$

(b)

Figure 1: Behavior of the Hubble parameter $H$ against time $t$ represents (a). (b) represents the behavior of the deceleration parameter $q$ against time $t$. The cosmological constant $\Lambda(t)$ is given by (6) and the potential $V(\phi)$ by (4).

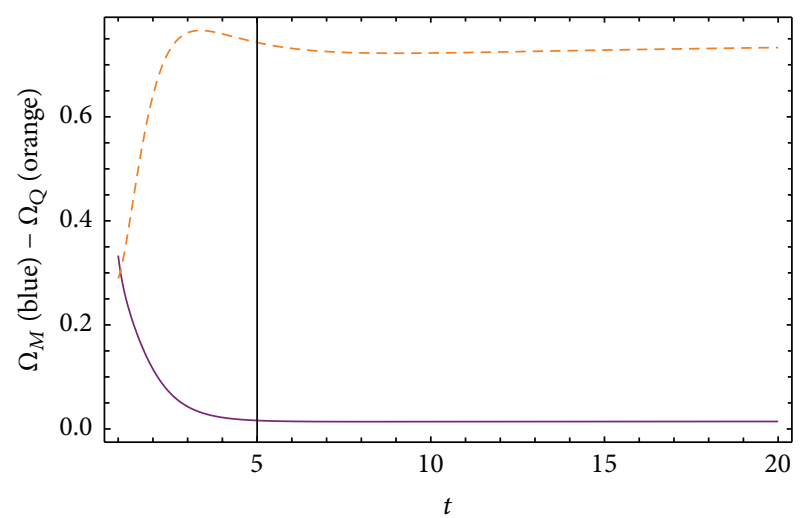

$$
\begin{aligned}
& \delta=0.3, \alpha=1 \\
& -\{\gamma=0.05, b=0.04, \omega=0.75\} \\
& ---\{\gamma=0.05, b=0.04, \omega=0.75\}
\end{aligned}
$$

(a)

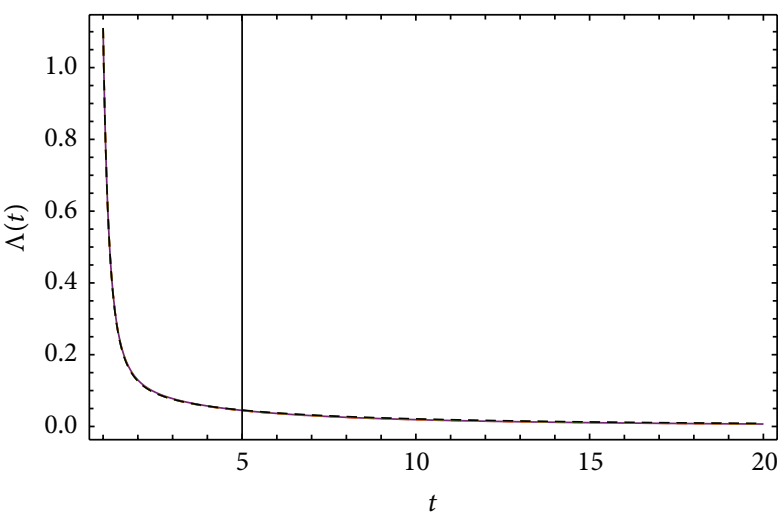

$$
\begin{aligned}
& \delta=0.3, \alpha=1 \\
& -\{\gamma=0, b=0, \omega=0.75\} \quad--\{\gamma=0.04, b=0.03, \omega=0.75\} \\
& --\{\gamma=0.02, b=0.01, \omega=0.75\}--\{\gamma=0.05, b=0.04, \omega=0.75\} \\
& --\{\{\gamma=0.03, b=0.02, \omega=0.75\}
\end{aligned}
$$

(b)

Figure 2: Behavior of the critical densities $\Omega_{\mathrm{Q}}$ and $\Omega_{M}$ of the DE and DM against time $t$ represents (a). (b) represents the behavior of the $\Lambda(t)$ against time $t$ for the model described via cosmological constant $\Lambda(t)$ given by (6) and the potential given by (4).

in the presence of the two other phenomenological forms of the $Q$ given as

$$
\begin{aligned}
& Q=3 H b \rho_{Q}+\gamma\left(\rho_{b}-\rho_{Q}\right) \frac{\dot{\phi}}{\phi}, \\
& Q=b H^{1-2 \gamma} \rho_{b}^{\gamma} \dot{\phi}^{2} .
\end{aligned}
$$

The interaction given by (23) was considered by us in GR with varying $G(t)$ and $\Lambda(t)$ recently in [51]. The second form of the interaction given via (24) was considered in [57]. Analysis reveals an interesting fact that all models having different forms of the interaction term $Q$ considered in this work reproduce the same behavior for the Universe and the cosmological parameters. The last part of this section is devoted to the cosmological model with $\Lambda=$ const. Consideration of the cosmological model with the constant $\Lambda$ allowed us to find that we have a transit Universe and with increasing value of the $\Lambda$ we will decrease the Hubble parameter $H$ and the deceleration parameter $q$ (Figure 4). In this case we are able to have the Universe which will enter the phase with $\omega_{\text {tot }}$ and $\omega_{\mathrm{Q}}$, to be very close to -1 but not exactly -1 (Figure 5 ). It is easy to see that the increasing $\Lambda$ will decrease cosmological parameters $\omega_{\mathrm{O}}$ and $\omega_{\text {tot }}$. In this case, we will have the old Universe which indicates 

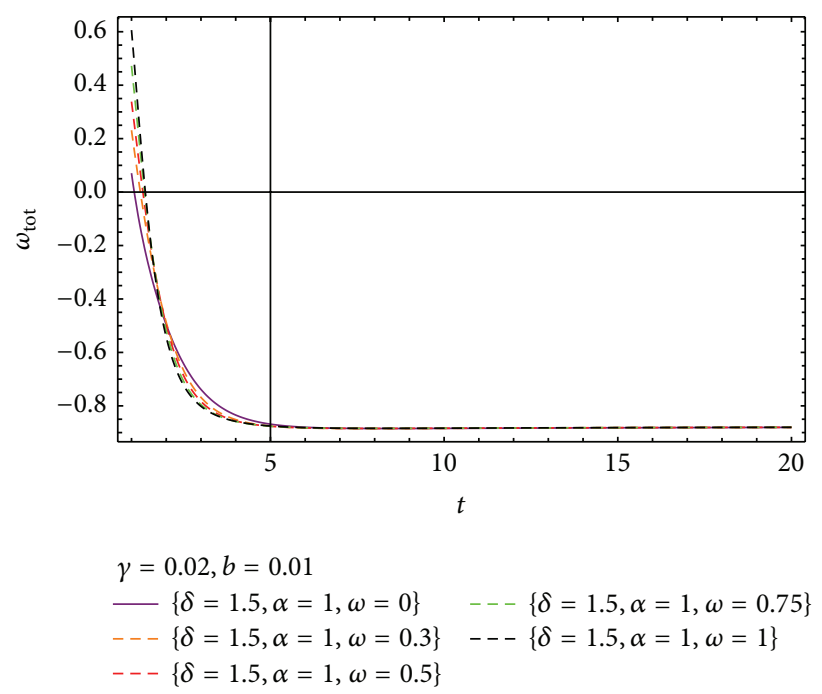

(a)

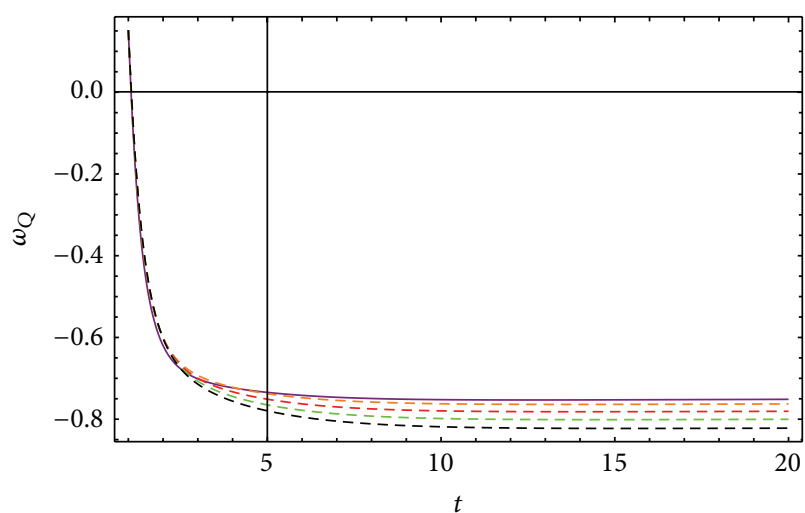

$$
\begin{aligned}
& \delta=0.3, \alpha=1 \\
& -\{\gamma=0, b=0, \omega=0.75\} \quad--\{\gamma=0.04, b=0.03, \omega=0.75\} \\
& --\{\gamma=0.02, b=0.01, \omega=0.75\}--\{\gamma=0.05, b=0.04, \omega=0.75\} \\
& --\{\gamma=0.03, b=0.02, \omega=0.75\}
\end{aligned}
$$

(b)

FIGURE 3: Behavior of the EoS parameter of the effective fluid $\omega_{\text {tot }}$ against time $t$ represents (a). (b) represents the behavior of the $\omega_{\mathrm{Q}}$ against time $t$ for the model described via cosmological constant $\Lambda(t)$ given by (6) and the potential given by (4).

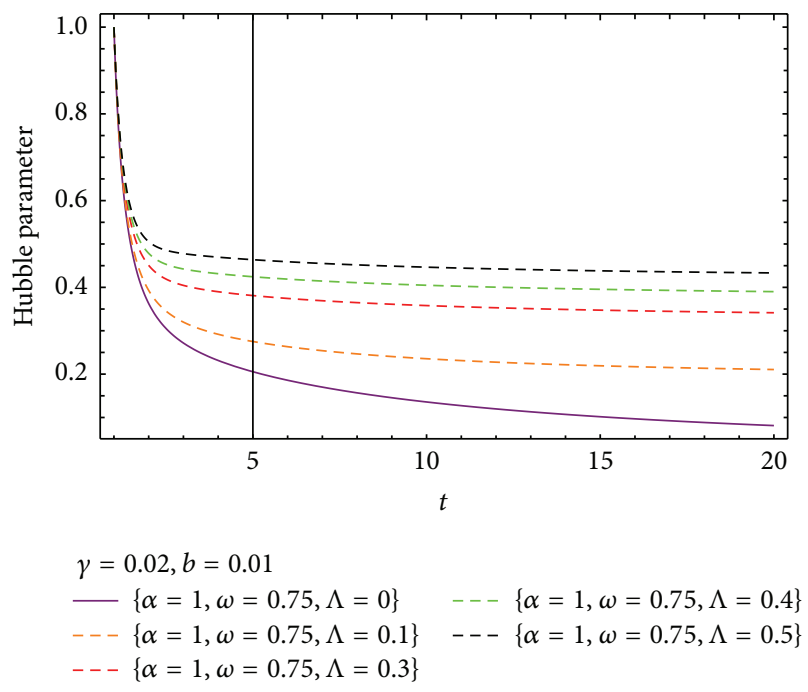

(a)

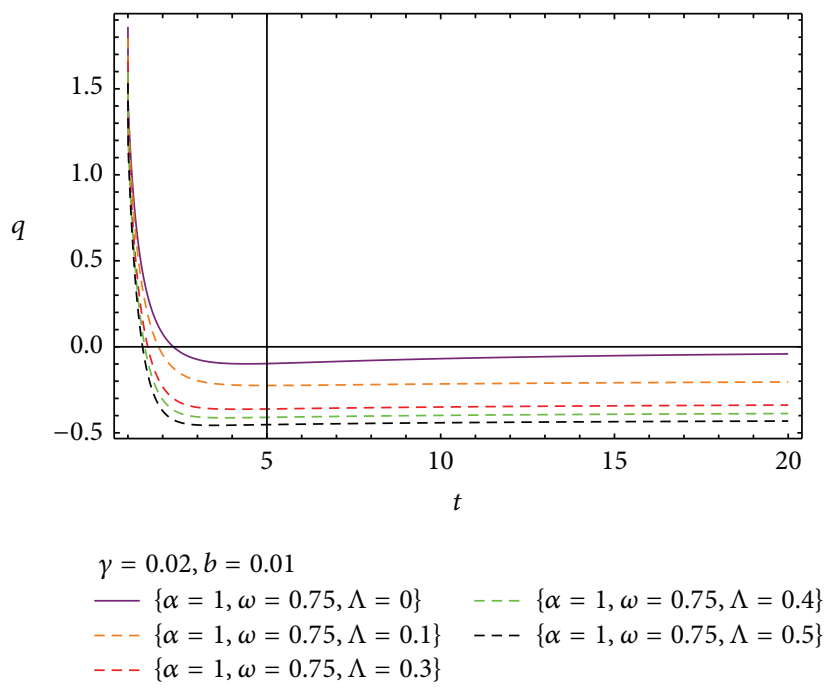

(b)

FIGURE 4: Behavior of the Hubble parameter $H$ against time $t$ represents (a). (b) represents the behavior of the deceleration parameter $q$ against time $t$. The potential $V(\phi)$ is given by (4) and $\Lambda=$ const.

accelerated expansion, where a very tiny amount of the barotropic fluid exists $\left(\Omega_{M} \neq 0\right)$; the dynamics of the Universe will be dominated by $\Lambda$ and DE only; because $\beta(t) \rightarrow 0$, increasing $\Omega_{\Lambda}$ will decrease $\Omega_{Q}$ which can be seen from Figure 6. Consideration of the other forms for the interaction term $Q$ given by (23)-(24) does not reveal significant changes in the model; therefore we decided to save a place and time and not to discuss the graphical behavior of the cosmological parameters for the other models. In the next section we would like to illuminate some physical aspects of the phenomenological model via the square of sound speed $C_{S}^{2}$ and observational constraints.

\section{4. $C_{S}^{2}$ and the Observational Constraints on the Model}

The simplest way to reject the cosmological model is to consider the behavior of the square of sound speed $C_{S}^{2}$. Today, the widespread opinion is to have the following constraint on it:

$$
0 \leq C_{S}^{2} \leq 1
$$

The criteria expressed in (25) indicate the bounds at which small perturbations of the background energy density propagate. It is possible to find argumentation challenging 


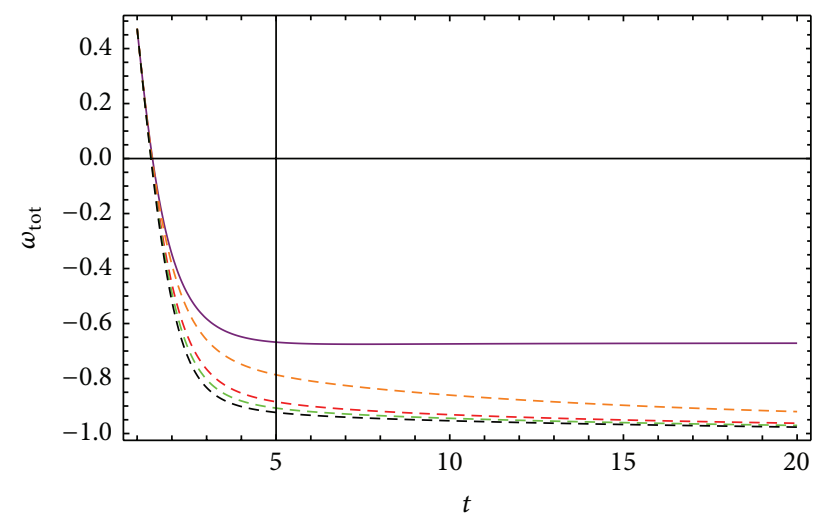

$$
\begin{array}{ll}
\gamma=0.02, b=0.01 & \\
-\{\alpha=1, \omega=0.75, \Lambda=0\} & --\{\alpha=1, \omega=0.75, \Lambda=0.4\} \\
---\{\alpha=1, \omega=0.75, \Lambda=0.1\} & ---\{\alpha=1, \omega=0.75, \Lambda=0.5\} \\
---\{\alpha=1, \omega=0.75, \Lambda=0.3\} &
\end{array}
$$

(a)

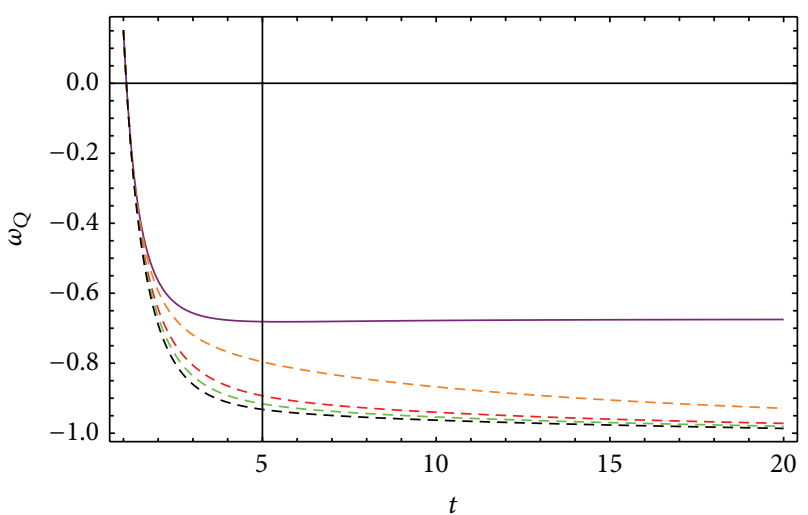

$$
\begin{array}{ll}
\gamma=0.02, b=0.01 & \\
-\{\alpha=1, \omega=0.75, \Lambda=0\} & --\{\alpha=1, \omega=0.75, \Lambda=0.4\} \\
---\{\alpha=1, \omega=0.75, \Lambda=0.1\} & ---\{\alpha=1, \omega=0.75, \Lambda=0.5\} \\
---\{\alpha=1, \omega=0.75, \Lambda=0.3\} &
\end{array}
$$

(b)

FIGURE 5: Behavior of the EoS parameter of the effective fluid $\omega_{\text {tot }}$ against time $t$ represents (a). (b) represents the behavior of the $\omega_{\mathrm{Q}}$ against time $t$. The potential $V(\phi)$ is given by (4) and $\Lambda=$ const.

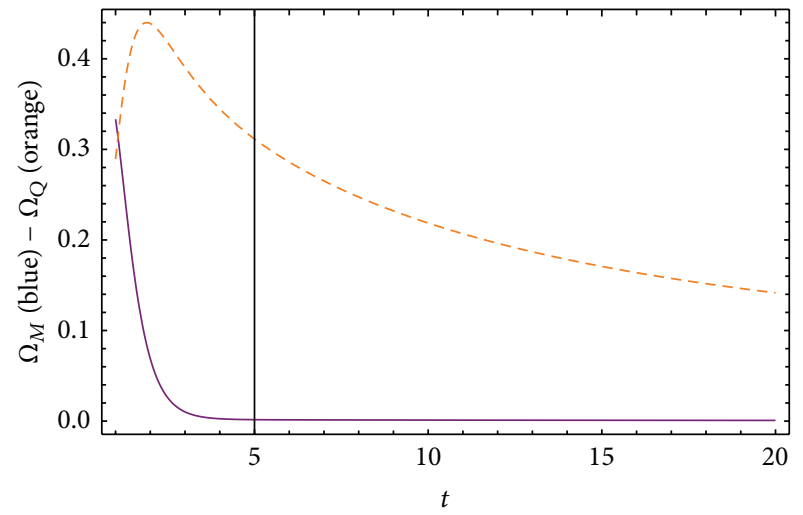

$$
\begin{aligned}
& \gamma=0.02, b=0.01 \\
& -\{\alpha=1, \omega=0.75, \Lambda=0.3\} \\
& --\{\alpha=1, \omega=0.75, \Lambda=0.3\}
\end{aligned}
$$

(a)

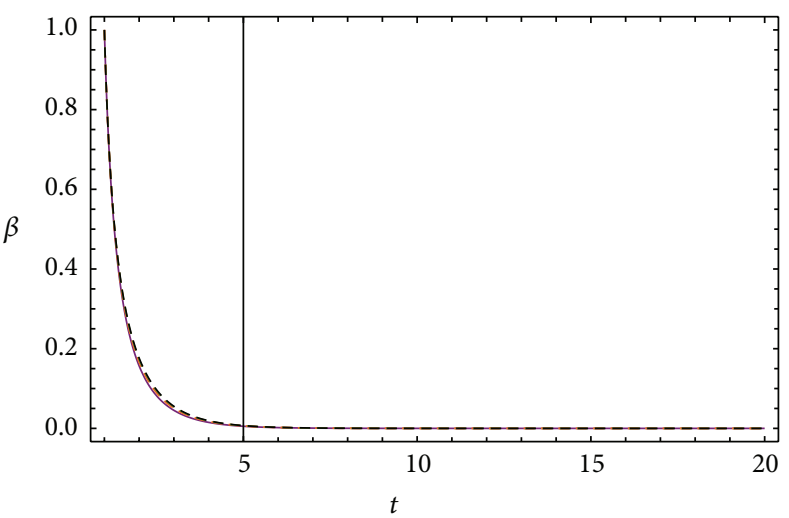

$$
\begin{aligned}
& \alpha=1, \gamma=0.03 \\
& -\{\omega=0, b=0.02, \Lambda=0.2\} \quad--\{\omega=0.6, b=0.02, \Lambda=0.2\} \\
& ---\{\omega=0.3, b=0.02, \Lambda=0.2\}--\{\{\omega=0.75, b=0.02, \Lambda=0.2\} \\
& --\{\omega=0.5, b=0.02, \Lambda=0.2\}
\end{aligned}
$$

FIGURE 6: Behavior of the critical densities $\Omega_{\mathrm{Q}}$ and $\Omega_{M}$ of the DE and DM against time $t$ represents (a). (b) represents the behavior of the $\beta(t)$ against time $t$. The potential $V(\phi)$ is given by (4) and $\Lambda=$ const.

the case of $C_{S}^{2}>1$ [58]. A negative $C_{S}^{2}$ will indicate that given perturbations are unstable. One should not discard the possibility of $C_{S}^{2}<0$ without careful investigation [59]. For our model in both cases with constant cosmological constant $\Lambda$ and varying cosmological constant $\Lambda(t)$, we found that, for the reasonable values of the parameters of the model obtained from the observational constraints, the $C_{S}^{2}$ for the $\mathrm{DE}$ and for the effective fluid are positive for the early stages of the evolution, while they are negative for the old Universe (Figure 7). The observational constraints on the models are obtained based on the SNIa test, which is based on the distance modulus $\mu$ (Figure 8 ) related to the luminosity distance $D_{L}$ by

$$
\mu=m-M=5 \log _{10} D_{L},
$$

where $D_{L}$ is defined as

$$
D_{L}=(1+z) \frac{c}{H_{0}} \int_{0}^{z} \frac{d z^{\prime}}{\sqrt{H\left(z^{\prime}\right)}} .
$$

The quantities $m$ and $M$ denote the apparent and the absolute magnitudes, respectively. There are many different SNIa 
TABLE 1

\begin{tabular}{lcccccc}
\hline$\gamma$ & $b$ & $\delta$ & $\alpha$ & $\omega$ & $H_{0}$ & $\Omega_{\mathrm{Q}}$ \\
\hline $0.03 \pm 0.03$ & $0.04 \pm 0.04$ & $1.5_{-0.7}^{+0.5}$ & $0.9_{-0.2}^{+0.3}$ & $0.5_{-0.4}^{+0.25}$ & $0.9_{-0.4}^{+0.3}$ & $0.8_{-0.2}^{+0.15}$ \\
\hline
\end{tabular}

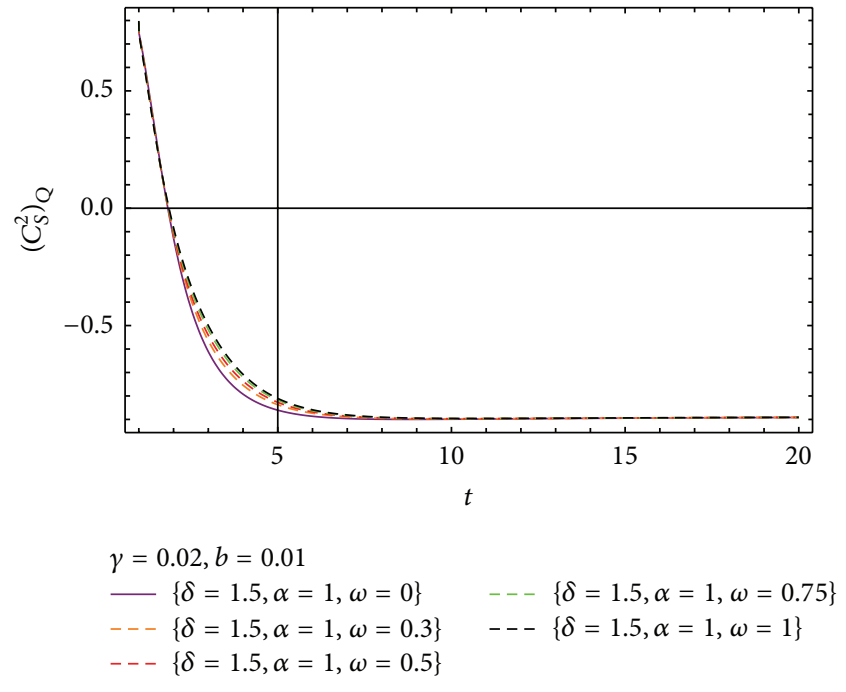

(a)

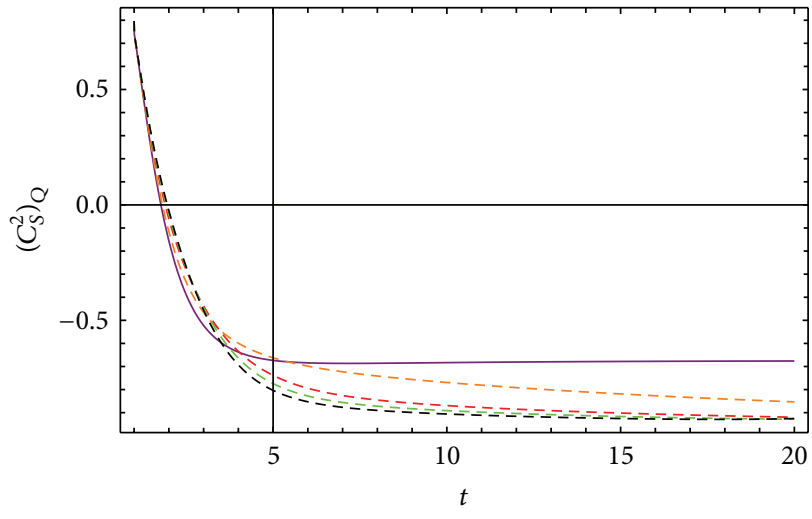

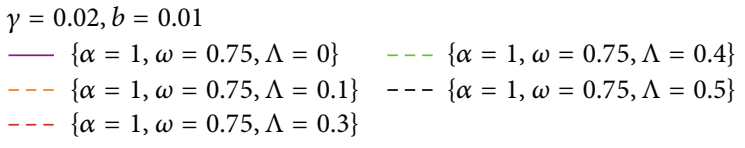

(b)

Figure 7: Behavior of the $C_{S}^{2}$ for the DE against time $t$ represents (a) with the potential $V(\phi)$ given by (4) for the varying $\Lambda(t)$ given by (6). (b) represents the behavior of the $C_{S}^{2}$ against time $t$ for the potential given by (4) and $\Lambda=$ const.

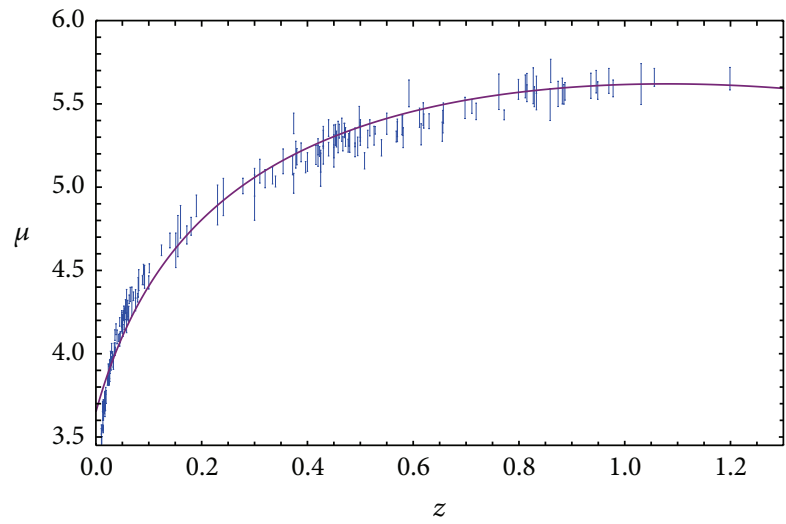

Figure 8: Observational data SNeIa + BAO + CMB for distance modulus versus our theoretical results.

data sets, obtained with different techniques. In some cases, these different samples may give very different results. Our observational analysis of the background dynamics uses the following three tests: the differential age of old objects based on the $H(z)$ dependence as well as the data from SNIa and from BAO. A fourth test could potentially be added: the position of the first peak of the anisotropy spectrum of the cosmic microwave background radiation (CMB). However, the $\mathrm{CMB}$ test implies integration of the background equations until $z \approx 1.000$ which requires the introduction of the radiative component.
But the inclusion of such radiative component considerably changes the structure of the equations and no analytic expression for $H(z)$ is available. Hence, we will limit ourselves to the mentioned three tests for which a reliable estimation is possible. We presented Table 1 containing information on the values of the parameters of the model with the varying cosmological constant $\Lambda(t)$ obtained from the observational constraints.

In the next section we will provide discussion of the obtained results to conclude our work and finalize the paper.

\section{Discussion}

In this work we proposed and considered cosmological models involving interacting quintessence DE on the Lyra manifold, which gives an interesting modification of the field equations. Moreover, the cosmology with a varying cosmological constant has been assumed. In our model we tried to make a connection between the Hubble parameter $H$, the scalar field $\phi$, and the potential $V(\phi)$ to obtain the varying cosmological constant. Given form of the interaction term $Q=3 H b \rho+\gamma \dot{\rho}$ between the $\mathrm{DE}$ and a barotropic fluid results in a transit Universe, where the deceleration parameter $q$ changes the sign from the positive to negative, independently whether we consider constant cosmological constant or a varying cosmological constant $\Lambda(t)$ under consideration. Analysis of the critical densities of the components reveals that in the initial Universe the barotropic fluid was dominant over the DE (for some values of the model parameters this 
domination can be neglected), which quickly changes during the evolution. The cosmological model with varying $\Lambda(t)$ indicates that for the later stages of the evolution our Universe will enter the phase where $\mathrm{DE}$ will dominate in the dynamics of the Universe where $\Omega, \beta(t)$, and $\Lambda(t)$ will accept tiny small positive values. This means that with this case the evolution of the Universe from the early times to the future will be described by the theory differing from GR. We should note that the two quantities $\Lambda(t)$ and $\beta(t)$ are decreasing functions over time, which also corporates with the physical theories perfectly. With the constant cosmological constant we have rather simplified scenario, because we got $\beta(t) \rightarrow$ 0 immediately, when $a(t) \rightarrow \infty$, which means that the latter Universe will recover GR to describe its dynamics. We should note that a transit Universe is obtained and as was expected increasing $\Lambda$ will suppress the domination of the $\mathrm{DE}$ in the old Universe, where $\Omega_{m} \rightarrow 0$. In both cases the EoS parameters of the effective fluid and the DE remain strictly above -1 indicating the quintessence Universe with the true quintessence DE. Causality, based on the "good" theories, implies restriction on the square of speed sound to be $0 \leq C_{S}^{2} \leq 1$, which is a simple and a good way to reject or accept cosmological models. However, as we mentioned in the main text, this restriction can be challenged with different arguments. Unfortunately, if we adhere to widespread opinion, then our models with $C_{S}^{2}<0$ for the later stages of the evolution, which indicates instability of the theory, should be rejected. Related to this, we assume that our model can represent a possible behavior of an early Universe only, because only there we are able to obtain $0 \leq C_{S}^{2} \leq$ 1 . With the observational constraints we found constraints on the parameters of the models. We have some analysis in the literature according to which with $1 \sigma$ level from $H(z)$ data we have $q \approx-0.3$ and $H_{0}=68.43 \pm 2.8(\mathrm{Km} / \mathrm{sMpc})$ [60]. On the other hand from data of SNeIa we have $q \approx$ -0.43 and $H_{0}=69.18 \pm 0.55(\mathrm{Km} / \mathrm{sMpc})$ [60]. Also joint test using $H(z)$ and SNeIa gives $-0.39 \leq q \leq-0.29$ and $H_{0}=68.93 \pm 0.53(\mathrm{Km} / \mathrm{sMpc})[60]$. Recent astronomical data based on a new infrared camera on the HST gives $H_{0}=73.8 \pm 2.4(\mathrm{Km} / \mathrm{sMpc})$ [61]. The other probe using galactic clusters data suggests $H_{0}=67 \pm 3.2(\mathrm{Km} / \mathrm{sMpc})$ [62]. Finally, $\Lambda \mathrm{CDM}$ model suggests $q \rightarrow-1$. Conclusion of the presented facts is that generally $q \geq-1$. We see that our models are able to reproduce the above-mentioned behavior for the Hubble parameter $H$ (in appropriate units) and the deceleration parameter $q$. Obtained behavior of the models encouraged us to extend models and as a first step we considered other forms of the interaction (mentioned in the main text), but we do not see any significant changes in the behavior of the Universe, which deserves to be included in this work. Our future interest in this direction is to consider cosmological models with different forms of the varying $\Lambda(t)$ on one hand, and on the other hand instead of the barotropic fluid consider different nonlinear EoS fluids, particularly, ones which include bulk viscosity. The last aspect is motivated by the fact that in an isotropic and homogeneous Universe bulk viscosity will model irreversible processes. Obtained results hopefully will be reported in forthcoming articles.

\section{Conflict of Interests}

The author declares that there is no conflict of interests regarding the publication of this paper.

\section{References}

[1] A. G. Riess, A. V. Filippenko, P. Challis et al., "Observational evidence from supernovae for an accelerating universe and a cosmological constant," The Astronomical Journal, vol. 116, no. 3, p. 1009, 1998.

[2] S. Perlmutter, G. Aldering, G. Goldhaber et al., "Measurements of $\Omega$ and $\Lambda$ from 42 high-redshift supernovae," The Astrophysical Journal, vol. 517, no. 2, p. 565, 1999.

[3] R. Amanullah, C. Lidman, D. Rubin et al., "Spectra and hubble space telescope light curves of six type ia supernovae at $0.511<$ $z<1.12$ and the union2 compilation," The Astrophysical Journal, vol. 716, p. 712, 2010.

[4] A. C. Pope, T. Matsubara, A. S. Szalay et al., "Cosmological parameters from eigenmode analysis of sloan digital sky survey galaxy redshifts," The Astrophysical Journal, vol. 607, no. 2, p. 655, 2004.

[5] D. N. Spergel, L. Verde, H. V. Peiris et al., "First-year Wilkinson Microwave Anisotropy Probe (WMAP)* observations: determination of cosmological parameters," The Astrophysical Journal Supplement Series, vol. 148, no. 1, p. 175, 2003.

[6] G. C. Samanta, "Bianchi type-III cosmological models with anisotropic dark energy (DE) in lyra geometry," International Journal of Theoretical Physics, vol. 52, no. 10, pp. 3442-3456, 2013.

[7] S. Weinberg, "The cosmological constant problems (Talk given at Dark Matter 2000, February, 2000)," http://arxiv.org/abs/ astro-ph/0005265.

[8] J. Solà and H. Štefančić, "Effective equation of state for dark energy: Mimicking quintessence and phantom energy through a variable $\Lambda$," Physics Letters B, vol. 624, no. 3-4, pp. 147-157, 2005.

[9] I. L. Shapiro and J. Solà, "On the possible running of the cosmological 'constant"' Physics Letters B, vol. 682, no. 1, pp. 105-113, 2009.

[10] C. Armendariz-Picon, V. Mukhanov, and P. J. Steinhardt, "Dynamical solution to the problem of a small cosmological constant and late-time cosmic acceleration," Physical Review Letters, vol. 85, no. 21, pp. 4438-4441, 2000.

[11] C. Armendariz-Picon and V. Mukhanov, "Essentials of $k$ essence," Physical Review D, vol. 63, no. 10, Article ID 103510, 2001.

[12] M. Khurshudyan, J. Sadeghi, M. Hakobyan, H. Farahani, and R. Myrzakulov, "Interaction between modified Chaplygin gas and ghost dark energy in the presence of extra dimensions," The European Physical Journal Plus, vol. 129, article 119, 2014.

[13] Y.-B. Wu, Y.-Y. Su, J.-B. Lu et al., "Effects of modified Chaplygin gas on structure formation in the universe," General Relativity and Gravitation, vol. 45, no. 7, pp. 1387-1402, 2013.

[14] E. O. Kahya, M. Khurshudyan, B. Pourhassan, R. Myrzakulov, and A. Pasqua, "Higher order corrections of the extended Chaplygin gas cosmology with varying $G$ and $\Lambda$," http://arxiv.org/ abs/1402.2592.

[15] J. Sadeghi, M. Khurshudyan, M. Hakobyan, and H. Farahani, "Hubble parameter corrected interactions in cosmology," Advances in High Energy Physics, vol. 2014, Article ID 129085, 16 pages, 2014. 
[16] J. Sadeghi, M. Khurshudyan, and H. Farahani, "Phenomenological varying modified chaplygin gas with variable $G$ and $\Lambda$ : toy models for our universe," http://arxiv.org/abs/1308.1819.

[17] M. Khurshudyan, "Interaction between generalized varying Chaplygin gas and Tachyonic fluid," http://arxiv.org/abs/1301 .1021 .

[18] J. Sadeghi, B. Pourhassan, and Z. Abbaspour Moghaddam, "Interacting entropy-corrected holographic dark energy and IR cut-off length," International Journal of Theoretical Physics, vol. 53, no. 1, pp. 125-135, 2014.

[19] U. Debnath, "Constraining the parameters of modified chaplygin gas in Einstein-Aether gravity," Advances in High Energy Physics, vol. 2014, Article ID 653630, 8 pages, 2014.

[20] H. Saadat and B. Pourhassan, "Viscous varying generalized Chaplygin gas with cosmological constant and space curvature," International Journal of Theoretical Physics, vol. 52, no. 10, pp. 3712-3720, 2013.

[21] M. Sharif and M. Azam, "Spherical thin-shell wormholes and modified chaplygin gas," Journal of Cosmology and Astroparticle Physics, vol. 2013, no. 5, article 25, 2013.

[22] B. Pourhassan, "Viscous modified cosmic chaplygin gas cosmology," International Journal of Modern Physics D, vol. 22, no. 9, Article ID 1350061, 2013.

[23] C. Uggla, "Global cosmological dynamics for the scalar field representation of the modified Chaplygin gas," Physical Review D, vol. 88, Article ID 064040, 2013.

[24] H. Saadat and B. Pourhassan, "FRW bulk viscous cosmology with modified cosmic Chaplygin gas," Astrophysics and Space Science, vol. 344, no. 1, pp. 237-241, 2013.

[25] R. Herrera, M. Olivares, and N. Videla, "Intermediategeneralized Chaplygin gas inflationary universe model," The European Physical Journal C, vol. 73, no. 1, p. 2295, 2013.

[26] H. Saadat and B. Pourhassan, "Effect of varying bulk viscosity on generalized chaplygin gas," International Journal of Theoretical Physics, vol. 53, no. 4, pp. 1168-1173, 2014.

[27] J. Wang, Y.-B. Wu, D. Wang, and W.-Q. Yang, "Extended analysis on new generalized Chaplygin gas," Chinese Physics Letters, vol. 26, no. 8, Article ID 089801, 2009.

[28] H. Saadat and B. Pourhassan, "FRW bulk viscous cosmology with modified Chaplygin gas in flat space," Astrophysics and Space Science, vol. 343, no. 2, pp. 783-786, 2013.

[29] T. P. Sotiriou and V. Faraoni, " $f(R)$ theories of gravity," Reviews of Modern Physics, vol. 82, no. 1, pp. 451-497, 2010.

[30] Y.-F. Cai, S.-H. Chen, J. B. Dent, S. Dutta, and E. N. Saridakis, "Matter bounce cosmology with the $f(T)$ gravity," Classical and Quantum Gravity, vol. 28, no. 21, Article ID 215011, 2011.

[31] J. Wang, Y.-B. Wu, Y.-X. Guo, W.-Q. Yang, and L. Wang, "Energy conditions and stability in generalized $\mathrm{f}(\mathrm{R})$ gravity with arbitrary coupling between matter and geometry," Physics Letters B, vol. 689, no. 4-5, pp. 133-138, 2010.

[32] Y.-B. Wu, Y.-Y. Zhao, R.-G. Cai, J.-B. Lu, J.-W. Lu, and X.-J. Gao, "Thermodynamic laws for generalized $f(R)$ gravity with curvature-matter coupling," Physics Letters B, vol. 717, no. 4-5, pp. 323-329, 2012.

[33] S. Nojiri and S. D. Odintsov, "Introduction to modified gravity and gravitational alternative for dark energy," International Journal of Geometric Methods in Modern Physics, vol. 4, no. 1, pp. 115-145, 2007.

[34] S. Nojiri and S. D. Odintsov, "Unified cosmic history in modified gravity: from $\mathrm{F}(\mathrm{R})$ theory to Lorentz non-invariant models," Physics Reports, vol. 505, no. 2-4, pp. 59-144, 2011.
[35] G. Cognola, E. Elizalde, S. Nojiri, S. D. Odintsov, L. Sebastiani, and S. Zerbini, "Class of viable modified $f(R)$ gravities describing inflation and the onset of accelerated expansion," Physical Review D, vol. 77, no. 4, Article ID 046009, 2008.

[36] A. V. Astashenok, S. Capozziello, and S. D. Odintsov, "Further stable neutron star models from $f(R)$ gravity," Journal of Cosmology and Astroparticle Physics, vol. 12, article 040, 2013.

[37] H. Farajollahi, J. Sadeghi, and M. Pourali, "Stability analysis of holographic dark energy in Brans-Dicke cosmology," Astrophysics and Space Science, vol. 341, no. 2, pp. 695-700, 2012.

[38] M. Khurshudyan, B. Pourhassan, and A. Pasqua, "Higher derivative corrections of $f(R)$ gravity with varying equation of state in the case of variable $G$ and $\Lambda$," http://arxiv.org/abs/1401 .6630 .

[39] B. Ratra and P. J. E. Peebles, "Cosmological consequences of a rolling homogeneous scalar field," Physical Review D, vol. 37, no. 12, pp. 3406-3427, 1988.

[40] C. Wetterich, "Cosmology and the fate of dilatation symmetry," Nuclear Physics, Section B, vol. 302, no. 4, pp. 668-696, 1988.

[41] A. R. Liddle and R. J. Scherrer, "Classification of scalar field potentials with cosmological scaling solutions," Physical Review D, vol. 59, Article ID 023509, 1998.

[42] I. Zlatev, L. Wang, and P. J. Steinhardt, "Quintessence, cosmic coincidence, and the cosmological constant," Physical Review Letters, vol. 82, no. 5, pp. 896-899, 1999.

[43] K. Guo, N. Ohta, and Y. Z. Zhang, "Parametrizations of the dark energy density and scalar potentials," Modern Physics Letters A, vol. 22, no. 12, pp. 883-890, 2007.

[44] S. Dutta, E. N. Saridakis, and R. J. Scherrer, "Dark energy from a quintessence (phantom) field rolling near a potential minimum (maximum)," Physical Review D, vol. 79, Article ID 103005, 2009.

[45] E. N. Saridakis and S. V. Sushkov, "Quintessence and phantom cosmology with nonminimal derivative coupling," Physical Review D, vol. 81, Article ID 083510, 2010.

[46] M. Khurshudyan, E. Chubaryan, and B. Pourhassan, "Interacting quintessence models of dark energy," International Journal of Theoretical Physics, vol. 53, no. 7, pp. 2370-2378, 2014.

[47] M. Khurshudyan, A. Pasqua, J. Sadeghi, and H. Farahani, "Quintessence cosmology with an effective $\Lambda$-term in Lyra manifold," http://arxiv.org/abs/1402.0118.

[48] W. Hao, "Cosmological constraints on the sign-changeable interactions," Communications in Theoretical Physics, vol. 56, no. 5, p. 972, 2011.

[49] P. G. Ferreira and M. Joyce, "Structure formation with a selftuning scalar field," Physical Review Letters, vol. 79, no. 24, pp. 4740-4743, 1997.

[50] E. J. Copeland, A. R. Liddle, and D. Wands, "Exponential potentials and cosmological scaling solutions," Physical Review $D$, vol. 57, no. 8, pp. 4686-4690, 1998.

[51] M. Khurshudyan, "Phenomenological models of universe with varying $G$ and $\Lambda$," Central European Journal of Physics, vol. 12, no. 5, pp. 348-366, 2014.

[52] G. Lyra, "Über eine modifikation der riemannschen geometrie," Mathematische Zeitschrift, vol. 54, pp. 52-64, 1951.

[53] D. K. Sen, "A static cosmological model," Zeitschrift für Physik, vol. 149, no. 3, pp. 311-323, 1957.

[54] K. A. Dunn, "A scalar-tensor theory of gravitation in a modified Riemannian manifold," Journal of Mathematical Physics, vol. 12, p. $578,1971$. 
[55] W. D. Halford, "Cosmological theory based on Lyra's geometry," Australian Journal of Physics, vol. 23, no. 5, pp. 863-870, 1970.

[56] V. K. Shchigolev, "Cosmology with an effective $\Lambda$-term in Lyra manifold," Chinese Physics Letters, vol. 30, Article ID 119801, 2013.

[57] X.-m. Chen, Y. Gong, and E. N. Saridakis, "Phase-space analysis of interacting phantom cosmology," Journal of Cosmology and Astroparticle Physics, vol. 2009, 2009.

[58] J. K. Erickson, R. R. Caldwell, P. J. Steinhardt, C. ArmendarizPicon, and V. Mukhanov, "Measuring the speed of sound of quintessence," Physical Review Letters, vol. 88, no. 12, Article ID 121301, 2002.

[59] T. Koivisto and D. F. Mota, "Dark energy anisotropic stress and large scale structure formation," Physical Review D, vol. 73, no. 8, Article ID 083502, 2006.

[60] S. Kumar, "Observational constraints on Hubble constant and deceleration parameter in power-law cosmology," Monthly Notices of the Royal Astronomical Society, vol. 422, no. 3, pp. 2532-2538, 2012.

[61] A. G. Riess, L. Macri, S. Casertano et al., "A 3\% solution: determination of the Hubble constant with the Hubble space telescope and wide field camera 3," The Astrophysical Journal, vol. 730, no. 2, p. 119, 2011.

[62] F. Beutler, C. Blake, M. Colless et al., "The 6dF Galaxy Survey: baryon acoustic oscillations and the local Hubble constant," Monthly Notices of the Royal Astronomical Society, vol. 416, no. 4, pp. 3017-3032, 2011. 

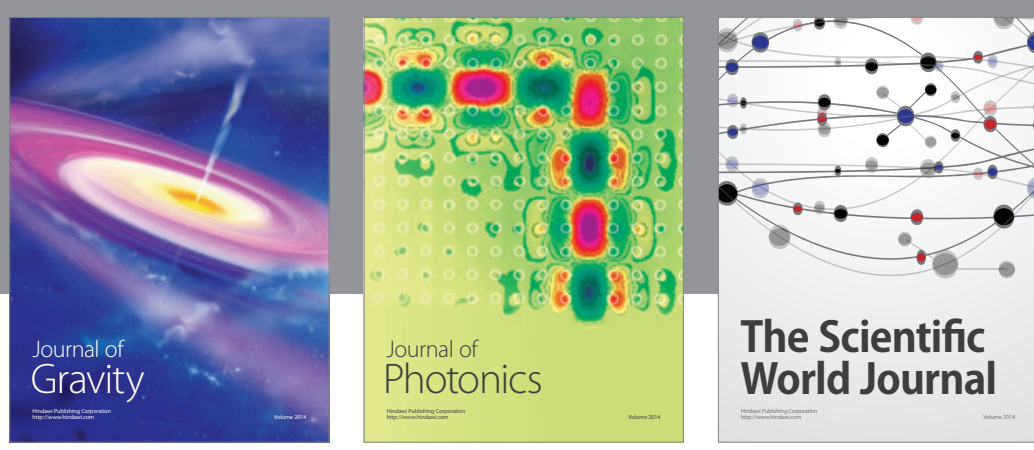

The Scientific World Journal
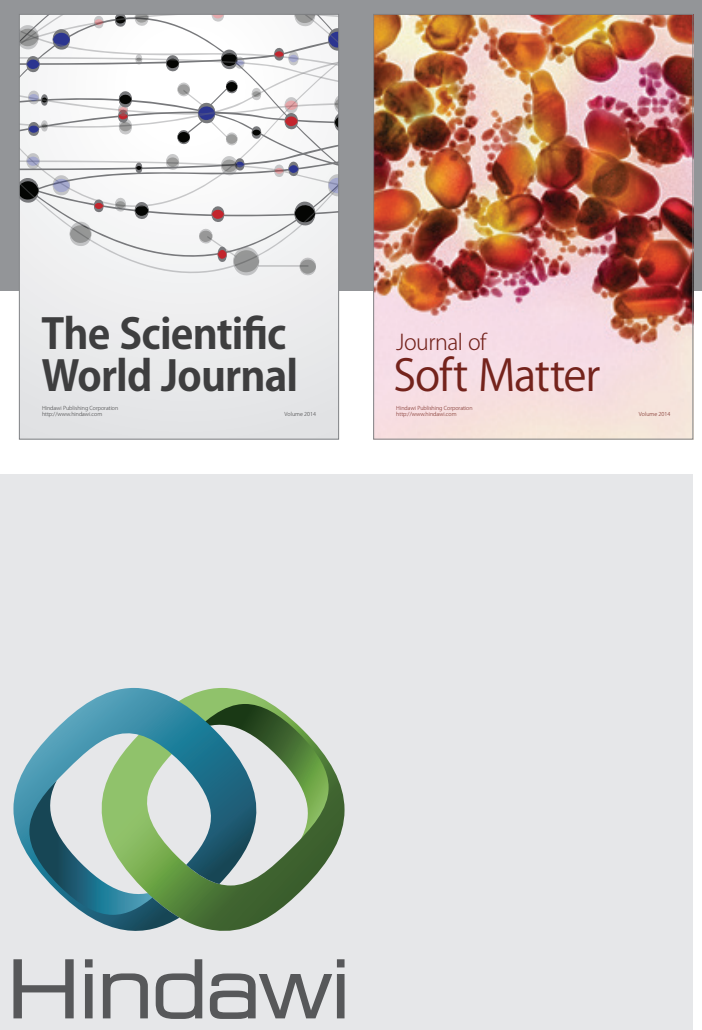

Submit your manuscripts at

http://www.hindawi.com

nternational Journal of

Statistical Mechanics
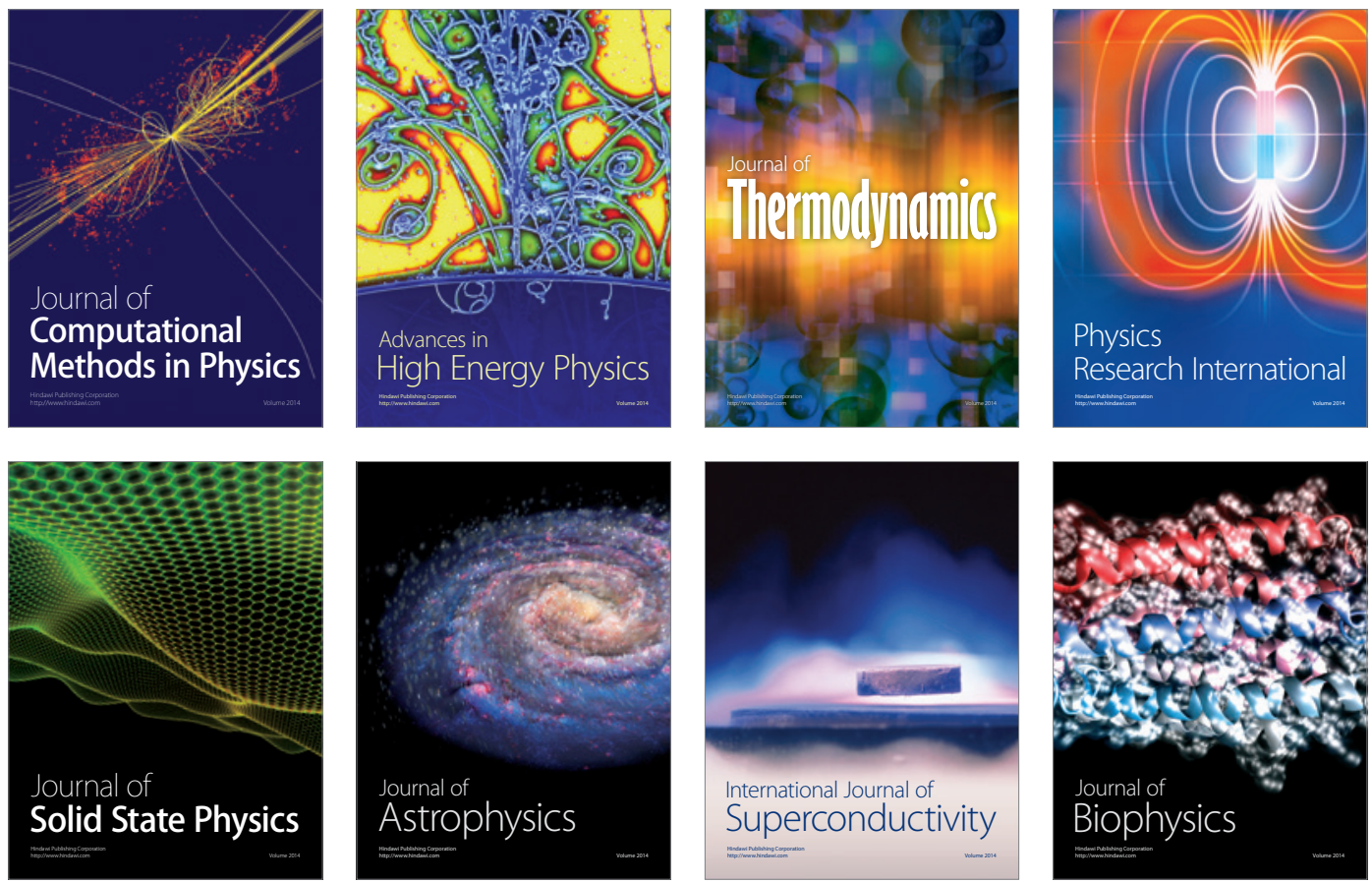
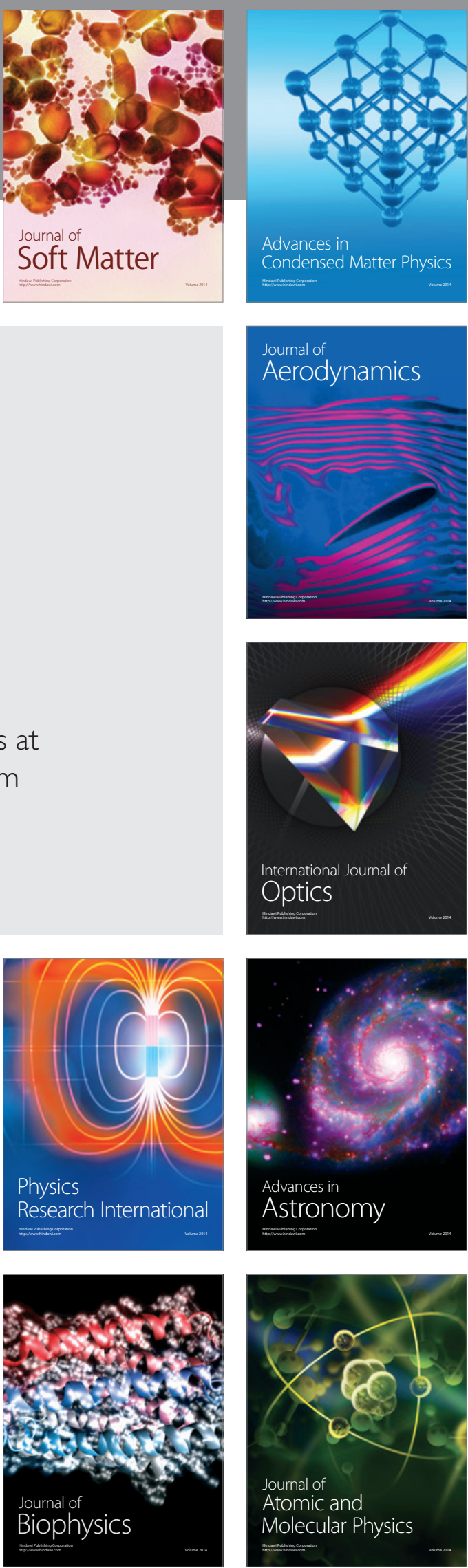